\title{
Model Reduction for Linear Descriptor Systems with Many Ports
}

\author{
Peter Benner and André Schneider
}

\begin{abstract}
Circuit simulation for power grid models leads to the challenge of model order reduction for linear descriptor systems with many ports. Based on the ESVDMOR idea of Feldmann and Liu [4], we have proposed several numerical improvements for ESVDMOR to enable the application to sparse and very large-scale systems. In further investigations we have proven that ESVDMOR is, under a few assumptions, stability, passivity, and reciprocity preserving. This paper provides a survey of these developments and outlines error estimation for ESVDMOR.
\end{abstract}

\section{Introduction}

Research in circuit simulation deals among others with linear parasitic systems which are, if they are in usual form, very suitable for model order reduction (MOR). Consequently, MOR became a standard tool over the last decades. Unfortunately, many known approaches are not able to handle a very special structure of today's systems, namely a large number of I/O-terminals. In recent years, this problem became a focus of numerous investigations. Especially from the industrial point of view, this problem needs to be solved as fast as possible to avoid a deadlock in process development due to a lack of simulation know-how. There is a basic idea of Feldmann and Liu [4], on which our work is based on. We modify the algorithm in a way such that it does not need expensive computational steps anymore, e.g. we replace a full SVD by a truncated one. Consequently, it becomes applicable for very large-scale linear continuous time-invariant systems up to order $n=10^{6}$, or even larger. Beyond that, we discuss questions about passivity, stability and reciprocity preservation, which are again very important for real world applications. Especially

Peter Benner, André Schneider

Max Planck Institute for Dynamics of Complex Technical Systems, Magdeburg, Germany, e-mail: benner@mpi-magdeburg.mpg.de schneidera@mpi-magdeburg.mpg.de 
reciprocity, i.e. the possibility of synthesizing the reduced model as a circuit in applications of circuit simulation, is a very important question. Often reduced order models are dense and not physically interpretable. Error analysis and industrial implementation are the last stages on the way to provide a useful and powerful tool to handle these special structures mentioned above. This paper gives an overview about the theoretical results. Due to space limitations, extensive numerical studies will be presented in a succeeding publication.

\section{Theoretical Properties of ESVDMOR}

Modeling of dynamical processes from various application areas, e.g. circuit simulation, mechanical constructs, and biological or biochemical reactions, leads to linear time-invariant continuous-time descriptor systems

$$
C \dot{x}(t)=-G x(t)+B u(t), \quad y(t)=L x(t), \quad x(0)=x_{0},
$$

with $C, G \in \mathbb{R}^{n \times n}, B \in \mathbb{R}^{n \times m_{\text {in }}}, L \in \mathbb{R}^{m_{\text {out }} \times n}, x(t) \in \mathbb{R}^{n}$ containing internal state variables, $u(t) \in \mathbb{R}^{m_{\text {in }}}$ the vector of inputs, $y(t) \in \mathbb{R}^{m_{\text {out }}}$ being the output vector, $x_{0} \in \mathbb{R}^{n}$ the initial value, $n$ the number of state variables, and the number of inputs $m_{i n}$, which is not necessarily equal to the number of outputs $m_{\text {out }}$. We assume the transfer function of (1) in the frequency domain to be

$$
H(s)=L(s C+G)^{-1} B .
$$

In this section we briefly discuss the basics of (E)SVDMOR for systems with $\mathscr{O}(n) \approx \mathscr{O}\left(m_{\text {in } / \text { out }}\right)$. In $[4,6]$ it is shown that it is possible to make use of inner system correlations regarding input and output terminals. Consider the $i$-th block moment of (2) as $\mathbf{m}_{\mathbf{i}}=L\left(-G^{-1} C\right)^{i} G^{-1} B, i=0,1, \ldots$, where $\mathbf{m}_{\mathbf{i}}$ is an $m_{\text {out }} \times m_{\text {in }}$ matrix. These moments are equal to the coefficients of the Taylor series expansion of (2) about $s_{0}=0, H(s)=\sum_{i=0}^{\infty} m_{i}(s)^{i}$. The expansion in $s=s_{0}$ leads to frequencyshifted moments

$$
\mathbf{m}_{\mathbf{i}}\left(s_{0}\right)=L\left(-\left(s_{0} C+G\right)^{-1} C\right)^{i}\left(s_{0} C+G\right)^{-1} B, \quad i=0,1, \ldots
$$

Thus, the Taylor series expansion including these moments is $H(s)=\sum_{i=0}^{\infty} m_{i}(s-$ $\left.s_{0}\right)^{i}$. We use $r$ different (frequency shifted) block moments to create the input response matrix $M_{I}$ and the output response matrix $M_{O}$, which are defined as:

$$
M_{I}=\left[\mathbf{m}_{\mathbf{0}}{ }^{T}, \mathbf{m}_{\mathbf{1}}{ }^{T}, \ldots, \mathbf{m}_{\mathbf{r}-\mathbf{1}}{ }^{T}\right]^{T}, \quad M_{O}=\left[\mathbf{m}_{\mathbf{0}}, \mathbf{m}_{\mathbf{1}}, \ldots, \mathbf{m}_{\mathbf{r}-\mathbf{1}}\right]^{T} .
$$

Note that if the number of rows in each matrix of (4) is not larger than the number of columns, then $r$ has to be increased. SVDMOR can be seen as a special case of ESVDMOR with $r=1$, i.e., only $m_{0}$ is used. Next, we apply the SVD to (4) in order to obtain a low rank approximation $M_{I}=U_{I} \Sigma_{I} V_{I}^{T} \approx U_{I_{r_{i}}} \Sigma_{I_{r_{i}}} V_{I_{r_{i}}}^{T}$, and $M_{O}=U_{O} \Sigma_{O} V_{O}^{T} \approx$ 
$U_{O_{r_{o}}} \Sigma_{O_{r_{o}}} V_{O_{r_{o}}}^{T}$, where $\Sigma_{I_{r_{i}}}$ and $\Sigma_{O_{r_{o}}}$ are $r_{i} \times r_{i}$ and $r_{o} \times r_{o}$ diagonal matrices, $V_{I_{r_{i}}}$ and $V_{O_{r_{o}}}$ are $m_{\text {in }} \times r_{i}$ and $m_{\text {out }} \times r_{o}$ isometric matrices that contain the dominant column subspaces of $M_{I}$ and $M_{O}$, and $U_{I_{r_{i}}}$ and $U_{O_{r_{o}}}$ are $r m_{\text {out }} \times r_{i}$ and $r m_{i n} \times r_{o}$ isometric matrices. They are not used any further. The values $r_{i} \leq m_{\text {in }}$ and $r_{o} \leq m_{\text {out }}$ denote the number of the virtual input and output terminals of the terminal reduced order model that are equal to the number of significant, i.e., not neglected singular values. The approximations of $B$ and $L$ using the matrices $V_{I_{r_{i}}}^{T}$ and $V_{O_{r_{o}}}^{T}$ lead to $B \approx B_{r} V_{I_{r_{i}}}^{T}$ and $L \approx V_{O_{r_{o}}} L_{r}$, where $B_{r} \in \mathbb{R}^{n \times r_{i}}$ and $L_{r} \in \mathbb{R}^{r_{o} \times n}$ are consequences of applying the Moore-Penrose pseudoinverse (denoted by $\left.(\cdot)^{+}\right)$of $V_{I_{r_{i}}}^{T}$ and $V_{O_{r_{o}}}$ (which are isometric) to $B$ and $L$, respectively. In detail, we have $B_{r}=B V_{I_{r_{i}}}\left(V_{I_{r_{i}}}^{T} V_{I_{r_{i}}}\right)^{-1}=B V_{I_{r_{i}}}^{T+}=B V_{I_{r_{i}}}$ and $L_{r}=\left(V_{O_{r_{o}}}^{T} V_{O_{r_{o}}}\right)^{-1} V_{O_{r_{o}}}^{T} L=V_{O_{r_{o}}}^{+} L=V_{O_{r_{o}}}^{T} L$, where $B_{r} \in \mathbb{R}^{n \times r_{i}}$ and $L_{r} \in \mathbb{R}^{r_{o} \times n}$. This leads to the desired decomposition of the transfer function

$$
H(s) \approx \widehat{H}(s)=V_{O_{r_{o}}} \underbrace{L_{r}(G+s C)^{-1} B_{r}}_{:=H_{r}(s)} V_{I_{r_{i}}}^{T},
$$

which is equivalent to a terminal reduction step. $V_{O_{r_{o}}}$ and $V_{I_{r_{i}}}^{T}$ can be understood as operators mapping the information from the original terminals to the virtual ones and back. The new inner transfer function $H_{r}(s)$, which has just a few virtual inputs and outputs, can be further reduced by means of any established MOR method, such that

$$
\tilde{H}_{r}(s)=\tilde{L}_{r}(\tilde{G}+s \tilde{C})^{-1} \tilde{B}_{r} \approx H_{r}(s) .
$$

Equation (5) is of essential matter for property preservation of the whole method, see Sec. 3. We end up with a very compact terminal reduced and reduced-order model $\tilde{H}_{r}(s)$ that approximates the original transfer function, i. e.

$$
H(s) \approx \widehat{H}(s)=V_{O_{r_{o}}} H_{r}(s) V_{I_{r_{i}}}^{T} \approx \widehat{H}_{r}(s)=V_{O_{r_{o}}} \tilde{H}_{r}(s) V_{I_{r_{i}}}^{T} .
$$

\section{Numerical Algorithm, Properties, and Error Estimation}

In this section, we briefly describe the numerical improvements we have implemented. Then we show preservation properties of the method and at the end we discuss error estimation for ESVDMOR.

The SVD is one of the crucial ingredients of the original idea. We forbear to perform a full SVD and neglect some of the singular triples simply because it is too expensive. Instead, we perform an efficient truncated SVD to calculate just the needed singular values (SV), i.e., SVs that are kept as well as SVs needed for error estimation. Additionally, we do not compute the moments in (3) explicitly but use an iterative application of matrix vector multiplication to factors of the moments. For illustration, we choose $M_{I}$ consisting of $r$ different moments. All presented approaches apply similarly to $M_{O}$. The singular triples of $M_{I}$ are computed 


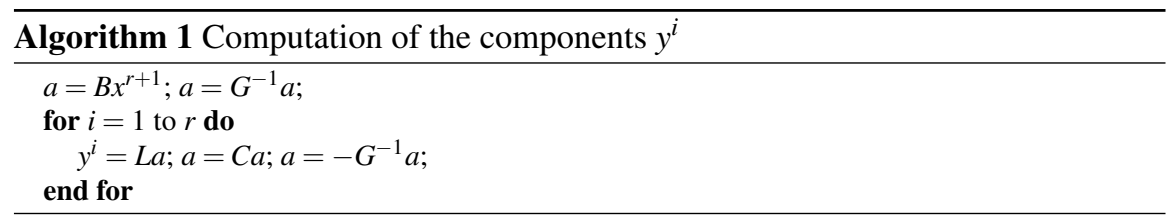

by solving an eigenvalue computation of the augmented matrix $A=\left(\begin{array}{cc}0 & M_{I} \\ M_{I}^{T} & 0\end{array}\right)$ using Krylov subspaces and matrix vector multiplication. The output of this multiplication is a vector $\mathbf{y} \in \mathbb{R}^{r \cdot m_{\text {out }}+m_{\text {in }}}$ of the same structure as the input vector $\mathbf{x}$, such that $A \mathbf{x}=: \mathbf{y}=\left(\left(y^{1}\right)^{T},\left(y^{2}\right)^{T}, \ldots,\left(y^{r+1}\right)^{T}\right)^{T}$, where $y^{i}=\left(y_{(i-1) \cdot m_{\text {out }}+1}, \ldots, y_{i \cdot m_{\text {out }}}\right)^{T}$ and $y^{r+1}=\left(y_{r \cdot m_{\text {out }}+1}, \ldots, y_{r \cdot m_{\text {out }}+m_{\text {in }}}\right)^{T}$, for $i=1, \ldots, r$. After matrix multiplication we get the components $y^{i}$ and $y^{r+1}$ of vector $\mathbf{y}$ as

$$
y^{i}=\mathbf{m}_{\mathbf{i}-\mathbf{1}} x^{r+1} \quad \text { and } \quad y^{r+1}=\mathbf{m}_{\mathbf{0}}^{T} x^{1}+\cdots+\mathbf{m}_{\mathbf{r}-\mathbf{1}}^{T} x^{r} .
$$

For efficiency reasons, we replace the block moments with their factors. We compute the $r+1$ parts of $\mathbf{y}$ by repeatedly applying the same factors to parts of $\mathbf{x}$, depending on whether it is a part of (7a) or (7b). The computation for (7a) follows Algorithm 1. The computation of (7b) is more involved, but follows the same recursive principle. For large $r$, there is a chance that numerical stability problems accrue, but in practice, $r$ is often small.

In the following, we summarize several facts on preservation of stability, passivity, and reciprocity in ESVDMOR reduced-order models. For detailed proofs, see [2]. Defining the descriptor system (1) as asymptotically stable if $\lim _{t \rightarrow \infty} x(t)=0$ for all solutions $x(t)$ of $C \dot{x}(t)=-G x(t)$, we have the following theorem:

Theorem 1. Consider an asymptotically stable system (1) with its transfer function (2). The ESVDMOR reduced-order system corresponding to (6) is asymptotically stable iff the inner reduction (5) is stability preserving.

A possible stability preserving model reduction method that can be applied along the lines of Theorem 1 is balanced truncation for regular descriptor systems, see [7]. Regarding passivity we note that a system is passive iff its transfer function is positive real [1]. The definition of positive realness can be found, e.g., in [5]. This definition requires $m_{\text {in }}=m_{\text {out }}=m$. If we assume $L=B^{T}$, such that $H(s)=B^{T}(s C+G)^{-1} B$ and

$$
\left[\begin{array}{cc}
C_{1} & 0 \\
0 & C_{2}
\end{array}\right] \dot{x}+\left[\begin{array}{cc}
G_{1} & G_{2} \\
-G_{2}^{T} & 0
\end{array}\right] x=\left[\begin{array}{c}
B_{1} \\
0
\end{array}\right] u, \quad y=\left[\begin{array}{ll}
B_{1} & 0
\end{array}\right] x,
$$

where $G_{1}, C_{1}, C_{2}$ are symmetric, $G_{1}, C_{1} \geq 0$ (i.e., both matrices are positive semidefinite), and $C_{2}>0$ (i.e., $C_{2}$ is positive definite), then $H(s)$ is positive real and thus the system is passive. This is a common structure among linear circuit models, see [5].

Theorem 2. Consider a passive system of the form (8). The ESVDMOR reduced system (6) is passive iff the inner reduction (5) is passivity preserving. 
Definition 1. A transfer function (2) is reciprocal if there exists $m_{1}, m_{2} \in \mathbb{N}$ with $m_{1}+m_{2}=m$, such that for $\Sigma_{e}=\operatorname{diag}\left(I_{m_{1}},-I_{m_{2}}\right)$ and all $s \in \mathbb{C}$, where $H(s)$ has no pole, it holds $H(s) \Sigma_{e}=\Sigma_{e} H^{T}(s)$. The matrix $\Sigma_{e}$ is called external signature of the system. A descriptor system is called reciprocal if its transfer function is reciprocal.

As a consequence, a transfer function of a reciprocal system has the form $H(s)=$ $\left[\begin{array}{cc}H_{11}(s) & H_{12}(s) \\ -H_{12}^{T}(s) & H_{22}(s)\end{array}\right]$, where $H_{11}(s)=H_{11}^{T}(s) \in \mathbb{R}^{m_{1}, m_{1}}$ and $H_{22}(s)=H_{22}^{T}(s) \in \mathbb{R}^{m_{2}, m_{2}}$.

Theorem 3. Consider a reciprocal system of the form (8). The ESVDMOR reduced system (6) is reciprocal iff the inner reduction (5) is reciprocity preserving.

Next, we discuss some ideas on how to get a global error bound for ESVDMOR. For details see [3]. The error caused by a SVD of $M_{I}$ is $e_{M_{I}}=\left\|M_{I}(r)-U_{I_{r_{i}}} \Sigma_{r_{i}}^{I} V_{I_{r_{i}}}^{T}\right\|_{2}=$ $\sigma_{r_{i}+1}^{I}$, where $\Sigma_{r_{i}}^{I}=\operatorname{diag}\left(\sigma_{1}^{I} \geq \ldots \geq \sigma_{r_{i}}^{I} \geq \sigma_{r_{i+1}}^{I} \geq \ldots \geq \sigma_{m_{i n}}^{I} \geq 0\right) \approx \Sigma_{r_{i}}^{I}=\operatorname{diag}\left(\sigma_{1}^{I} \geq\right.$ $\ldots \geq \sigma_{r_{i}}^{I}$ ). The error for the square root variant of balanced truncation is bounded by $\left\|H_{r}-\tilde{H}_{r}\right\|_{\mathscr{H}_{\infty}} \leq 2 \sum_{k=\ell+1}^{n} \hat{\sigma}_{k}=\delta$, in case we keep the $\ell$ largest $\hat{\sigma}_{i}$. Due to (6) and the triangle inequality, the total ESVDMOR error in spectral norm on the imaginary axis can be expressed locally as

$$
e_{\text {tot }}=\left\|H(i \omega)-\hat{H}_{r}(i \omega)\right\|_{2} \leq \underbrace{\|H(i \omega)-\hat{H}(i \omega)\|_{2}}_{=e_{\text {out }}}+\underbrace{\left\|\hat{H}(i \omega)-\hat{H}_{r}(i \omega)\right\|_{2}}_{e_{\text {in }}} .
$$

The error caused by the inner reduction follows from (6) and (9) as

$$
e_{i n}=\left\|V_{O_{r_{o}}} H_{r}(s) V_{I_{r_{i}}}^{T}-V_{O_{r_{o}}} \tilde{H}_{r}(s) V_{I_{r_{i}}}^{T}\right\|_{2}=\left\|H_{r}(s)-\tilde{H}_{r}(s)\right\|_{2} \leq \delta,
$$

due to the fact that the spectral norm is invariant under orthogonal transformations. The outer reduction error $e_{\text {out }}$ in the SVDMOR case is based on $M_{I}=M_{O}^{T}=m_{0}=$ $B^{T}\left(s_{0} C+G\right)^{-1} B=U \Sigma V^{T}=U \Sigma U^{T} \approx U_{r} \Sigma_{r} U_{r}^{T}$. The local terminal reduction error $e_{\text {out }}$ then is

$$
\begin{aligned}
e_{\text {out }} & =\left\|H\left(s_{0}\right)-\hat{H}\left(s_{0}\right)\right\|_{2} \\
\stackrel{(\mathrm{UNV})}{=} & \left\|B^{T}\left(s_{0} C+G\right)^{-1} B-U_{r} U_{r}^{T} B^{T}\left(s_{0} C+G\right)^{-1} B U_{r} U_{r}^{T}\right\|_{2} \stackrel{(\mathrm{SVD})}{=} \sigma_{k+1}^{I / O},
\end{aligned}
$$

if we keep $k$ singular values or terminals. Then the total error in the SVDMOR case in spectral norm is

$$
e_{t o t} \leq \sigma_{k+1}^{I / O}+2 \sum_{j=l+1}^{n} \hat{\sigma}_{j}
$$

For the ESVDMOR case with $r \geq 1$ ( $r$ times $m_{i}$ within the ansatz matrices), see [3]. 


\section{Conclusions}

This work gives an overview of the ESVDMOR approach with which, in combination with the right choice of the method in (5), it is possible to preserve stability, passivity, and reciprocity. Additionally, the possibility of a global error bound is given in(10). Despite the industrial need for such algorithms, very large-scale real world examples are hard to come by due to confidentiality. We have successfully reduced an academic state space example of order $10^{5}$ with originally circa half as much I/O-terminals and we have investigated an industrial circuit model of order $10^{3}$ with a few hundred pins. In any case, just as in standard MOR methods, the approaches are very dependent on the decay of the SVs. Furthermore, the reduced order model should be evaluated iteratively and in factorized form. Otherwise, ESVDMOR would be inefficient and we might end up with a very large-scale dense model due to the mapping back to the original terminals. With respect to the given hints, ESVDMOR is a powerful tool to reduce linear descriptor systems with many terminals.

Acknowledgements The work reported in this paper was supported by the German Federal Ministry of Education and Research (BMBF), grant no. 03BEPAE1. Responsibility for the contents of this publication rests with the authors.

\section{References}

1. Anderson, B., Vongpanitlerd, S.: Network Analysis and Synthesis. Prentice Hall, Englewood Cliffs, New Jersey, USA (1973)

2. Benner, P., Schneider, A.: On stability, passivity, and reciprocity preservation of ESVDMOR. In: P. Benner, M. Hinze, J. ter Maten (eds.) Model Reduction for Circuit Simulation, Lecture Notes in Electrical Engineering, vol. 74, pp. 267-278. Springer-Verlag, Berlin/Heidelberg, Germany (2011)

3. Benner, P., Schneider, A.: Some remarks on a priori error estimation for ESVDMOR. In: Scientific Computing in Electrical Engineering SCEE 2010 (2011). To appear

4. Feldmann, P., Liu, F.: Sparse and efficient reduced order modeling of linear subcircuits with large number of terminals. In: ICCAD '04: Proceedings of the 2004 IEEE/ACM Intl. Conf. Computer-aided design, pp. 88-92. IEEE Computer Society, Washington, DC, USA (2004)

5. Freund, R.W.: On Padé-type model order reduction of J-Hermitian linear dynamical systems. Linear Algebra and its Applications 429(10), 2451 - 2464 (2008)

6. Liu, P., Tan, S.X.D., Yan, B., McGaughy, B.: An efficient terminal and model order reduction algorithm. Integr. VLSI J. 41(2), 210-218 (2008)

7. Mehrmann, V., Stykel, T.: Balanced truncation model reduction for large-scale systems in descriptor form. In: P. Benner, V. Mehrmann, D. Sorensen (eds.) Dimension Reduction of LargeScale Systems, Lecture Notes in Computational Science and Engineering, vol. 45, pp. 83-115. Springer-Verlag, Berlin/Heidelberg, Germany (2005) 